\title{
Characterization of the quinolone resistant determining regions in clinical isolates of pneumococci collected in Canada
}

\author{
Samir N Patel ${ }^{1,2,3}$, Roberto Melano ${ }^{1,2,3}$, Allison McGeer ${ }^{1,2}$, Karen Green², Donald E Low ${ }^{1,2,3^{*}}$
}

\begin{abstract}
Background: The objective of this study was to examine Streptococcus pneumoniae isolates collected from a longitudinal surveillance program in order to determine their susceptibility to currently used fluoroquinolones and of the frequency and type of mutations in the quinolone-resistant determining regions (QRDRs) of their parC and gyrA genes.

Methods: The Canadian Bacterial Surveillance Network has been collecting clinical isolates of S. pneumoniae from across Canada since 1988. Broth microdilution susceptibility testing was carried out according to the Clinical and Laboratory Standards Institute guidelines. The QRDRs of the parC and gyrA genes were sequenced for all isolates with ciprofloxacin MIC $\geq 4 \mathrm{mg} / \mathrm{L}$, and a large representative sample of isolates $(\mathrm{N}=4,243)$ with $\mathrm{MIC} \leq 2 \mathrm{mg} / \mathrm{L}$.

Results: A total of 4,798 out of 30,111 isolates collected from 1988, and 1993 to 2007 were studied. Of those isolates that were successfully sequenced, 184 out of 1,032 with mutations in parC only, 11 out of 30 with mutations in gyrA only, and 292 out of 298 with mutations in parC and gyrA were considered resistant to ciprofloxacin (MIC $\geq 4 \mathrm{mg} / \mathrm{L})$. The most common substitutions in the parC were at positions $137(\mathrm{n}=722), 79(\mathrm{n}=$ 209), and 83 ( $n=56$ ), of which substitutions at positions 79 and 83 were associated with 4-fold increase in MIC to ciprofloxacin, whereas substitutions at position 137 had minimal effect on the ciprofloxacin MIC. A total of 400 out of 622 isolates with Lys-137 parC mutation belonged to serotypes 1, 12, 31, 7A, 9V, 9N and 9L, whereas only 49 out of 3064 isolates with no mutations belonged to these serotypes. Twenty-one out of 30 isolates with substitutions at position 81 of the gyrA gene had an increased MIC to ciprofloxacin. Finally, we found that isolates with mutations in both parC and gyrA were significantly associated with increased MIC to fluoroquinolones.
\end{abstract}

Conclusions: Not all mutations, most frequently Lys-137, found in the QRDRs of the parC gene of S. pneumoniae is associated with an increased MIC to fluoroquinolones. The high prevalence of Lys-137 appears to be due to its frequent occurrence in common serotypes.

\section{Background}

Streptococcus pneumoniae is a common cause of acute exacerbation of chronic bronchitis, sinusitis, and community-acquired pneumonia [1-3]. Treatment of respiratory tract infections has relied primarily on the use of $\beta$ lactam and macrolide antimicrobials; however in recent years the emergence of resistance to these agents has led to the widespread use of fluoroquinolones for adult patients with respiratory disease [4-7].

\footnotetext{
* Correspondence: dlow@mtsinai.on.ca

'Department of Microbiology, Mount Sinai Hospital, Toronto, Ontario, M5G
} $1 \times 5$, Canada
From the mid 1980s until the later part of the 1990s, earlier generation fluoroquinolones such as ciprofloxacin and ofloxacin were widely used for treatment of respiratory tract infections, despite having less than optimal activity against pneumococci. However, since the late 1990s, levofloxacin and moxifloxacin, with much greater in vitro activity and more optimal pharmacokinetic and pharmacodynamic properties for the treatment of pneumococcal infections, have replaced these agents $[8,9]$.

Fluoroquinolones target one of the two type II topoisomerases of pneumococci, DNA gyrase (composed of GyrA and GyrB subunit) and/or topoisomerase IV (composed of ParC and ParE subunits), resulting in 
inhibition of DNA synthesis [10-12]. Decreased susceptibility of S. pneumoniae to the fluoroquinolones has been attributed to either chromosomal mutations in the quinolone resistance-determining regions (QRDRs) of the $\operatorname{parC}$ and/or $g y r A$ genes, which encode for subunits of topoisomerase IV and DNA gyrase, respectively, and/ or by a reserpine-sensitive efflux mechanism [13-16]. Fluoroquinolones have binding preferences (primacy) for one of the enzymes found in the two type II topoisomerases. Primacy is determined most reliably by in vitro selection experiments in which a resistance mutation is observed first in one of the two genes of the primary target and gene mutations in the secondary target are only observed subsequently [17]. Mutant selection studies in S. pneumoniae have shown that the primary target for fluoroquinolones, such as ciprofloxacin, ofloxacin and levofloxacin, is topoisomerase IV. In contrast, newer fluoroquinolones such as moxifloxacin, gatifloxacin and gemifloxacin primarily target DNA gyrase $[18,19]$. In fact, gemifloxacin and moxifloxacin have been found in some reports to exhibit dual activity based on the minimal effect of either gyrA or parC mutations on resistance, whereas strains with mutations in both $\operatorname{gyr} A$ and $\operatorname{parC}$ exhibit high-level resistance to these agents [20-22].

Previous studies have shown that pneumococci with substitutions at positions 79 and 83 of $\operatorname{parC}$, and 81 and 85 of $g y r A$ are most frequently found in isolates with reduced susceptibility to fluoroquinolones [23-32]. However, many of these studies only sequenced isolates that were resistant to fluoroquinolones, were smaller in sample size, and had been done in a time before the widespread use of the newer generation fluoroquinolones. The purpose of this study was to characterize a large collection of contemporary isolates of pneumococci in order to identify mutations in the QRDR of their parC and $g y r A$ genes, and to determine the effect of these mutations on fluoroquinolone activity.

\section{Materials and methods}

The Canadian Bacterial Surveillance Network is comprised of more than 100 private laboratories and community and university-affiliated hospitals in all 10 provinces and 2 of 3 territories in Canada. In 1988, and in each year from 1993 to 2008, participating laboratories were asked, based on their size, to collect either the first 20 (for small laboratories) or the first 100 (for larger laboratories) consecutive clinical isolates, and then all sterile site isolates of S. pneumoniae. The date of collection, the source of the specimen, and the patient's age and sex were recorded on a standard form. Duplicate isolates from the same patient were excluded. Isolates were transported on chocolate agar slants or swabs to a central laboratory where they were confirmed to be $S$. pneumoniae by standard methodology and stored at $-70^{\circ} \mathrm{C}$. Prior to susceptibility testing, isolates were thawed and subcultured onto blood agar twice. Isolates were serotyped on the basis of capsular polysaccharide antigens by the quelling reactions [33]. In vitro broth microdilution susceptibility testing was performed and interpreted according to Clinical and Laboratory Standards Institute (CLSI) guidelines [34,35]. Isolates were classified as non-susceptible to an antimicrobial if their MICs were in either the intermediate or resistant category, and resistant to ciprofloxacin if their MIC was $\geq 4 \mathrm{mg} / \mathrm{L}$.

\section{Amplification and sequencing of QRDRs}

The QRDRs of the parC and gyrA genes were amplified using following primers: for parC (forward: CGGTTCAACGCCGTATTCTT, reverse: ATCCCAGTCGAACCATTGAC; 394 bp), gyrA (forward: TGTTCACCGTCGCATTCTCT, reverse: ATACCAGTTGCTCCATTAACC, 393 bp). Amplicons were sequenced at Agencourt Bioscience Corp. (Beverly, MA). Multiple nucleotide sequences were performed with the ClustalW2 program http://www.ebi.ac.uk/Tools/clustalw2/index.html. Both $\operatorname{parC}$ and $\operatorname{gyr} A$ genes of isolates with a ciprofloxacin MIC $\geq 4 \mathrm{mg} / \mathrm{L}$, a levofloxacin MIC $\geq 4 \mathrm{mg} / \mathrm{L}$ or a moxifloxacin $\mathrm{MIC} \geq 0.5 \mathrm{mg} / \mathrm{L}$ were amplified and sequenced $(\mathrm{N}=540)$. In addition, a $45 \%$ sample of isolates $(\mathrm{N}=2,102)$ with a ciprofloxacin MIC $=2 \mathrm{mg} / \mathrm{L}$, or a levofloxacin MIC of $=2 \mathrm{mg} / \mathrm{L}$ and or a moxifloxacin MIC $=0.25 \mathrm{mg} / \mathrm{L}$, and a $12 \%$ sample of other isolates $(\mathrm{N}=27,469)$ were also amplified and sequenced.

\section{Statistical Analysis}

Differences in group proportions were assessed with the chi square test for trend or fisher's exact test.

\section{Results and Discussion}

A total of 30,111 isolates were collected from 1988, and 1993 to 2007. Of these isolates, 11,403 (37.9\%) were recovered from a sterile site $(10,565$ from blood, 371 from CSF, and 467 from other sterile sites), and 18,601 (61.8\%) were from non-sterile sites (9,694 from sputum, 4,732 from eye, 1,988 from ear, 1,053 from bronchial washings, and 1,134 from other non-sterile sites). Overall, 534 out of $30,111(1.8 \%)$ of isolates were resistant to ciprofloxacin (MIC $\geq 4 \mathrm{mg} / \mathrm{L}) ; 360(1.2 \%)$ were non-susceptible to levofloxacin (MIC $\geq 4 \mathrm{mg} / \mathrm{L}$ ), and $283(1.1 \%)$ were non-susceptible to moxifloxacin (MIC $\geq 2 \mathrm{mg} / \mathrm{L}$ ).

We selected 4,798 isolates for molecular characterization of parC and gyrA genes. Of these, 517 out of 534 isolates with ciprofloxacin MIC $\geq 4 \mathrm{mg} / \mathrm{L}, 968$ out of 968 isolates with MIC $=2 \mathrm{mg} / \mathrm{L}$, and 3,267 out of 3,296 isolates with $\mathrm{MIC}<2 \mathrm{mg} / \mathrm{L}$ were successfully sequenced. As shown in Table 1, 1,032 isolates contained at least one mutation in parC, 30 isolates 
contained at least one mutation in $g y r A$, and 298 isolates contained at least one mutation in both parC and $g y r A$. Of those parC only mutant isolates, $17.8 \%$ were resistant to ciprofloxacin, $2.2 \%$ were non-susceptible to levofloxacin, and $0.4 \%$ to moxifloxacin. Among gyrA only mutant isolates, $36.7 \%$ were resistant to ciprofloxacin, $36.7 \%$ and $20 \%$ non-susceptible to levofloxacin and moxifloxacin, respectively. Finally, among isolates containing mutations in both parC and gyrA, 98.0\% were resistant to ciprofloxacin, $97.6 \%$ non-susceptible to levofloxacin, and $86.2 \%$ to moxifloxacin (Table 1 ).

Among isolates with parC only mutations, the most common mutations were at positions 137 (Lys-137) (N $=722,70.0 \%), 79($ Ser-79) $(\mathrm{N}=209,20.2 \%)$, and 83 (Asp-83) $(\mathrm{N}=56,5.4 \%)$ (Table 2$)$. The most common substitutions observed were Lys137Asn, Ser79Phe or Tyr, and Asp83Tyr or Asn. As shown in figure 1A, substitutions at both positions 79 (Ser-79) and 83 (Asp-83) were associated with four-fold increase in MIC to ciprofloxacin $(\mathrm{P}<0.0001)$. In contrast, substitution at position 137 (Lys-137) was not associated with increased MIC to ciprofloxacin when compared to isolates with no mutations in parC (Figure 1A). These data are consistent with previous studies in which isolates with mutations that resulted in Ser-79 or Asp-83 amino acid changes were associated with decreased susceptibility to the fluoroquinolones $[25,26,28,31]$. Other mutations were found much less commonly. Those occurring at position 78 (Asp-78), and possibly those at positions 52 (Ser-52), 91 (Asn-91), 115 (Ala-115) and 129 (Tyr-129) could be associated with increase MIC to ciprofloxacin, though statistical analyses were not done due to small number of isolates (Table 2). The presence of Ser-79 and Asp-83 mutations were associated with an approximately two fold increase in levofloxacin and moxifloxacin MICs, though none of the isolates with an amino acid change a position 83 , and only $1.6 \%$ and $4.3 \%$ of isolates with Ser-79 mutations were non-susceptible to moxifloxacin and levofloxacin, respectively (data not shown). However, it is important to note that the classification of non-susceptibility is based on CLSI breakpoints. S. pneumoniae can just as readily develop resistance to fluoroquinolones during therapy when a first-step mutation has occurred that results in reduced susceptibility to the fluoroquinolones, even though the increase in MIC does not meet CLSI criteria for nonsusceptibility. The combination of two mutations, each of which causes a reduction in the activity of the fluoroquinolone, results in a resistant strain [36,37].

We found that not all mutations resulted in a reduction of the activity of the fluoroquinolones. An amino acid change at Lys-137 in combination with a change in either Ser-79 or Asp-83did not significantly increased the ciprofloxacin MIC compared to isolates with Ser-79 or Asp-83 only changes (Ser-79 only vs Ser-79 + Lys137: $\mathrm{P}=0.330$; Asp-83 only vs Asp-83 + Lys-137: $\mathrm{P}=$ $0.5511)$. These data are consistent with previously reported studies in which a mutation at parC resulting in an amino acid change at Lys-137 did not play a significant role in increasing the MIC to fluoroquinolones [30,32].

If the Lys-137 parC mutation has only a marginal effect, if any, on fluoroquinolone activity, what would explain its presence and the frequency with which has been identified by others [38]? Its occurrence may just be the result of a polymorphism in $\operatorname{par} C$, similar to what has been seen in Streptococcus pyogenes [39] or as yet some unidentified fitness advantage. The frequency

Table 1 In vitro activities of fluoroquinolones against S. pneumoniae isolates with mutations in the parC and gyrA genes.

Minimum Inhibitory Concentration (mg/L) Number of Isolates

\begin{tabular}{|c|c|c|c|c|c|c|c|c|c|c|c|c|c|c|c|}
\hline & 0.015 & 0.03 & 0.06 & 0.12 & 0.25 & 0.5 & 1 & 2 & 4 & 8 & 16 & 32 & 64 & 128 & Total \\
\hline \multicolumn{16}{|l|}{ parC only } \\
\hline Ciprofloxacin & 0 & 0 & 0 & 0 & 2 & 182 & 408 & 256 & 136 & 39 & 5 & 2 & 2 & 0 & 1032 \\
\hline Levofloxacin & 0 & 0 & 1 & 0 & 0 & 205 & 580 & 213 & 15 & 3 & 4 & 1 & 0 & 0 & 1022 \\
\hline Moxifloxacin & 0 & 5 & 130 & 489 & 343 & 16 & 8 & 2 & 1 & 1 & 0 & 0 & 0 & 0 & 995 \\
\hline \multicolumn{16}{|l|}{ gyrA only } \\
\hline Ciprofloxacin & 0 & 0 & 0 & 0 & 0 & 1 & 9 & 9 & 2 & 5 & 2 & 2 & 0 & 0 & 30 \\
\hline Levofloxacin & 0 & 0 & 0 & 0 & 0 & 1 & 13 & 5 & 3 & 4 & 4 & 0 & 0 & 0 & 30 \\
\hline Moxifloxacin & 0 & 0 & 1 & 6 & 8 & 4 & 5 & 4 & 2 & 0 & 0 & 0 & 0 & 0 & 30 \\
\hline \multicolumn{16}{|l|}{ parC \& gyrA } \\
\hline Ciprofloxacin & 0 & 0 & 0 & 0 & 0 & 0 & 3 & 3 & 4 & 29 & 73 & 126 & 55 & 5 & 298 \\
\hline Levofloxacin & 0 & 0 & 0 & 0 & 0 & 1 & 6 & 0 & 13 & 78 & 151 & 37 & 11 & 1 & 298 \\
\hline Moxifloxacin & 0 & 0 & 0 & 4 & 6 & 3 & 26 & 136 & 87 & 18 & 1 & 1 & 0 & 0 & 282 \\
\hline
\end{tabular}




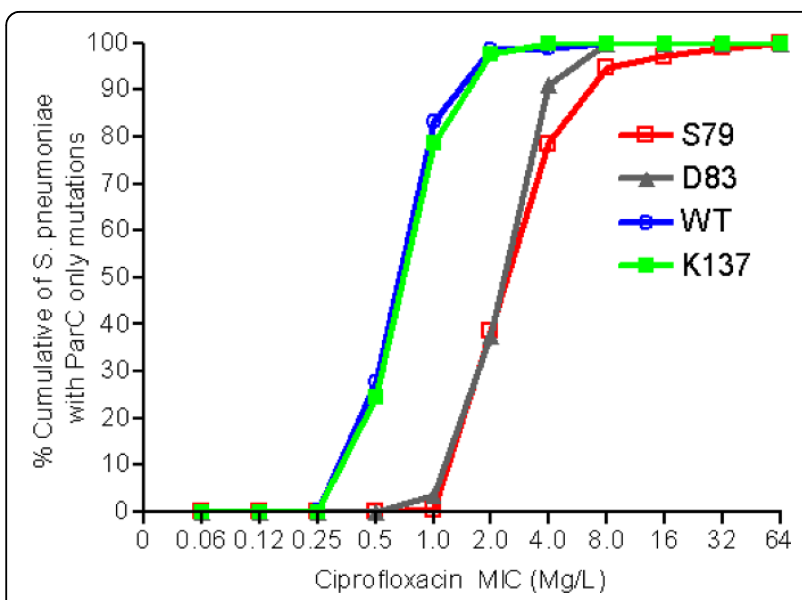

Figure 1 Distribution of ciprofloxacin MICs in S. pneumoniae isolates with a parC only amino acid substitutions. The figure shows that pneumococcal isolates with substitution at either position 79 or 83 of parC have four-fold increased ciprofloxacin MIC compared to isolates with no mutations in parC. On the other hand, substitution at position 137 of parC does not appear to increase ciprofloxacin MIC

with which it has been found is due in part to its association with specific common serotypes. We found that 400 out of 622 (64.3\%) isolates with Lys-137 parC mutation belonged to serotypes $1,12,31,7 \mathrm{~A}, 9 \mathrm{~V}, 9 \mathrm{~N}$ and $9 \mathrm{~L}$, whereas only 49 out of 3064 (1.6\%) of isolates with no mutations belonged to these serotypes. Among these serotypes, $9 \mathrm{~V}, 9 \mathrm{~N}$ and $9 \mathrm{~L}$ contained the majority of isolates with Lys-137 parC mutation (285 out of 400).

In Canada, there has been a sustained yearly increase in the use of the fluoroquinolones for the treatment of respiratory tract infections in adults during the last decade [6]. This has been accounted for in large part by the increased use of levofloxacin and moxifloxacin [6].
Despite the increased use of fluoroquinolones such as moxifloxacin that preferentially target GyrA, the number of mutations in the QRDR of the gyrA have gene remained low [40]. We found only 30 out of 3297 $(0.9 \%)$ isolates containing mutations in $g y r A$ only that resulted in an amino acid change in GyrA, with the most common substitution observed at position 81 (Ser81) (70\%; 21 out of 30) (Data not shown). The most common substitutions observed were Ser81Phe or Tyr in $g y r A$. The presence of a mutation in gyrA that resulted in an amino acid change at position 81 was associated with an approximately two fold increase in MIC to ciprofloxacin with $52.4 \%$ (11 out of 21 ) of isolates with this mutation resistant to ciprofloxacin. These data suggest that Ser-81 mutation in gyrA significantly reduces susceptibility to all fluoroquinolones.

Among isolates with mutations in both parC and gyrA, majority of these isolates carried amino acid alterations at Ser-79 and Ser-81 of parC and gyrA, respectively ( 214 of $298 ; 71.8 \%$ ), followed by amino acid alterations at Ser-79 and Glu-85 (45 of 298; $15.1 \%$ ). As shown in figure 2, isolates with mutations Ser-79 in parC and Ser-81 in gyrA were significantly associated with higher ciprofloxacin MIC with 69\% (145 out of 210 ) isolates had $\mathrm{MIC} \geq 32 \mathrm{mg} / \mathrm{L}$. Similarly, isolates containing mutations Ser-79 in parC and Glu-85 in gyrA, or Asp-83 in parC and Ser-81 in $g y r A$ were also significantly associated with increased MIC to ciprofloxacin. Interestingly, we had found 4 isolates containing mutations Lys-137 in parC and Ser-81 in gyrA, all of which were resistant to ciprofloxacin. However, MIC values were similar to the strains with mutations in gyrA ser-81 only, supporting the fact that mutation in $\operatorname{par} C$ Lys-137 is not associated with a reduction in fluoroquinolone activity.

Table 2 In vitro activity of ciprofloxacin against S. pneumoniae with mutations in the parC gene only.

\begin{tabular}{ccccccccccccc}
\hline & \multicolumn{10}{c}{ Ciprofloxacin } & $\begin{array}{c}\text { Minimum Inhibitory Concentration (mg/L) } \\
\text { Number of Isolates }\end{array}$ \\
\hline Substitution & $\mathbf{0 . 1 2}$ & $\mathbf{0 . 2 5}$ & $\mathbf{0 . 5}$ & $\mathbf{1}$ & $\mathbf{2}$ & $\mathbf{4}$ & $\mathbf{8}$ & $\mathbf{1 6}$ & $\mathbf{3 2}$ & $\mathbf{6 4}$ & Total \\
\hline None & 1 & 6 & 919 & 1,863 & 523 & 37 & 2 & 2 & 0 & 1 & 3,354 \\
\hline Asp-78 & 0 & 0 & 0 & 0 & 4 & 2 & 0 & 0 & 0 & 0 & 6 \\
\hline Asp-83 & 0 & 0 & 0 & 2 & 19 & 30 & 5 & 0 & 0 & 0 & 56 \\
\hline Lys-137 & 0 & 1 & 176 & 391 & 137 & 16 & 1 & 0 & 0 & 0 & 722 \\
\hline Arg-95 & 0 & 1 & 2 & 2 & 0 & 1 & 0 & 0 & 0 & 0 & 6 \\
\hline Ser-52 & 0 & 0 & 1 & 3 & 4 & 1 & 0 & 0 & 0 & 0 \\
\hline Ser-79 & 0 & 0 & 0 & 1 & 82 & 84 & 33 & 5 & 2 & 2 \\
\hline Other* & 0 & 0 & 3 & 6 & 7 & 1 & 0 & 0 & 0 & 0 \\
\hline Tyr-129 & 0 & 0 & 0 & 3 & 3 & 1 & 0 & 0 & 0 & 0 \\
\hline
\end{tabular}

*Mutations at other sites include: Ala-63 (1), Val-66 (2), Met-85 (1), Asn-91 (2) Glu-99 (1), Glu-100 (1), Gly-106 (1), Ser-107 (1), Met-108 (1), Ala-115 (2), Tyr-118 (1), Arg-122 (1), Leu-130 (1), Glu-135 (1), Thr-138 (1), Ala-158 (1). Of these, two (one with Asn-91-Asp mutation, and one with Ala-115-Pro mutation) had ciprofloxacin MICs of $4 \mathrm{mg} / \mathrm{L}$, and one (Thr-138-lle) had a levofloxacin MIC of $2 \mathrm{mg} / \mathrm{L}$. All others had MIC to ciprofloxacin of $<4 \mathrm{mg} / \mathrm{L}$, to levofloxacin of $<2 \mathrm{mg} / \mathrm{L}$, and to moxifloxacin of $<0.5 \mathrm{mg} / \mathrm{L})$ 


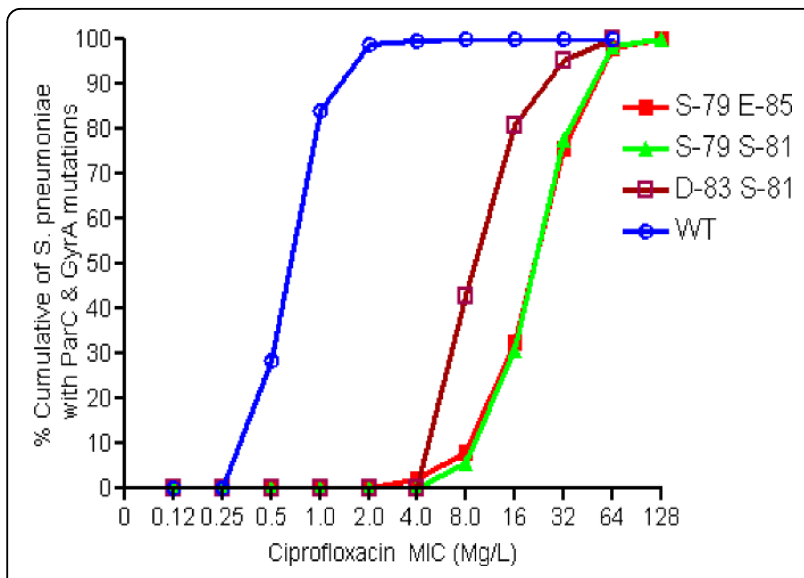

Figure 2 Distribution of pneumococcal isolates with amino acid substitutions in parC and gyrA against ciprofloxacin MIC.

The figure shows that isolates with amino acid substitutions at positions 79 of parC and 81 of gyrA or at positions 79 of parC and 85 of gyrA have 32 -fold higher ciprofloxacin MIC compared to isolates with no mutation in parC and gyrA. Similarly, substitutions at positions 83 of parC and 81 of gyrA result in 16-fold increase in ciprofloxacin MIC.

\section{Conclusion}

From this study of a large contemporary collection of pneumococci, we have found that, despite the widespread use of the fluoroquinolones for the treatment of respiratory tract infections in adults that resistance remains less the two percent. Also, despite the increase use of the C-8 methoxy fluoroquinolones including moxifloxacin and gatifloxacin which primarily target the type II topoisomerase GyrA, isolates with only mutations in the QRDRs of the gyrA only gene remain infrequent. Not all mutations, most frequently Lys-137, found in the QRDRs of the parC gene of S. pneumoniae is associated with an increased MIC to fluoroquinolones. The high prevalence of Lys-137 appears to be due to its frequent occurrence in common serotypes.

\section{Acknowledgements}

We are indebted to all of the hospitals and laboratories participating in the Canadian Bacterial Surveillance Network. This study was funded through the National Centre of Excellence in Bacterial Diseases, and by unrestricted educational grant from Bayer.

\section{Author details}

${ }^{1}$ Department of Microbiology, Mount Sinai Hospital, Toronto, Ontario, M5G 1X5, Canada. ${ }^{2}$ Department of Laboratory Medicine and Pathobiology, University of Toronto, Toronto, Ontario, M5G 1L5, Canada. ${ }^{3}$ Ontario Agency for Health Protection and Promotion, Toronto, Ontario, M9P 3T1, Canada.

\section{Authors' contributions}

SNP carried out statistical analysis of the data and drafted the manuscript. RM sequenced the isolates and helped in drafting the manuscript. AM participated in the design of the study and in the preparation of the manuscript. KG was involved in creation of the database to include all information regarding each isolate. DEL participated in the design and in the coordination of the study, and in the preparation of the manuscript. All authors read and approved the final manuscript.

\section{Competing interests}

SNP, RM and KG have none to declare. AM has served on speaker's bureau, and on advisory boards of Bayer and Wyeth. She has also received unrestricted investigator grants from Bayer and Wyeth. DEL is a member of Advanced Life Sciences and Boehringer Ingelheim USA Corporation advisory board committees. He has also consulted with MPM Asset management LLC., and has received research support from Advanced Life Sciences, Cerexa, Inc, GlaxoSmithKline, Inc., and Bayer Healthcare AG.

Received: 29 September 2009

Accepted: 18 January 2010 Published: 18 January 2010

\section{References}

1. Loeb M: Pneumonia in older persons. Clin Infect Dis 2003, 37(10):1335-1339.

2. Musher DM: Infections caused by Streptococcus pneumoniae: clinical spectrum, pathogenesis, immunity, and treatment. Clin Infect Dis 1992, 14(4):801-807.

3. Kadioglu A, Weiser JN, Paton JC, Andrew PW: The role of Streptococcus pneumoniae virulence factors in host respiratory colonization and disease. Nat Rev Microbiol 2008, 6(4):288-301.

4. Mandell LA, Wunderink RG, Anzueto A, Bartlett JG, Campbell GD, Dean NC, Dowell SF, File TM Jr, Musher DM, Niederman MS, Torres A, Whitney CG, Infectious Diseases Society of America; American Thoracic Society: Infectious Diseases Society of America/American Thoracic Society consensus guidelines on the management of community-acquired pneumonia in adults. Clin Infect Dis 2007, 44(Suppl 2):S27-72.

5. Fuller JD, McGeer A, Low DE: Drug-resistant pneumococcal pneumonia: clinical relevance and approach to management. Eur J Clin Microbiol Infect Dis 2005, 24(12):780-788.

6. Adam HJ, Hoban DJ, Gin AS, Zhanel GG: Association between fluoroquinolone usage and a dramatic rise in ciprofloxacin-resistant Streptococcus pneumoniae in Canada, 1997-2006. Int J Antimicrob Agents 2009, 34(1):82-85.

7. Patel SN, McGeer A, Green K, Low DE, Canadian Bacterial Surveillance Network (CBSN): Lack of emergence of gyrA mutations in S. pneumoniae (SP) despite increased fluoroquinolone (FQ) usage. 48th Annual Interscience Conference on Antimicrobial Agents and Chemotherapy/Infectious Disease of Society of America 46th Annual Meeting: 2008; Washington, DC, (Abstract \# C2-1843).

8. Shams WE, Evans ME: Guide to selection of fluoroquinolones in patients with lower respiratory tract infections. Drugs 2005, 65(7):949-991.

9. Ferrara AM: New fluoroquinolones in lower respiratory tract infections and emerging patterns of pneumococcal resistance. Infection 2005, 33(3):106-114.

10. Bast DJ, Low DE, Duncan CL, Kilburn L, Mandell LA, Davidson RJ, de Azavedo JC: Fluoroquinolone resistance in clinical isolates of Streptococcus pneumoniae: contributions of type II topoisomerase mutations and efflux to levels of resistance. Antimicrob Agents Chemother 2000, 44(11):3049-3054.

11. Bush K, Goldschmidt R: Effectiveness of fluoroquinolones against grampositive bacteria. Curr Opin Investig Drugs 2000, 1(1):22-30.

12. Heaton VJ, Ambler JE, Fisher LM: Potent antipneumococcal activity of gemifloxacin is associated with dual targeting of gyrase and topoisomerase IV, an in vivo target preference for gyrase, and enhanced stabilization of cleavable complexes in vitro. Antimicrob Agents Chemother 2000, 44(11):3112-3117.

13. Van Bambeke F, Michot JM, Van Eldere J, Tulkens PM: Quinolones in 2005: an update. Clin Microbiol Infect 2005, 11(4):256-280.

14. Hawkey PM: Mechanisms of quinolone action and microbial response. $J$ Antimicrob Chemother 2003, 51(Suppl 1):29-35.

15. Smith HJ, Nichol KA, Hoban DJ, Zhanel GG: Dual activity of fluoroquinolones against Streptococcus pneumoniae: the facts behind the claims. J Antimicrob Chemother 2002, 49(6):893-895.

16. Pestova E, Millichap JJ, Noskin GA, Peterson LR: Intracellular targets of moxifloxacin: a comparison with other fluoroquinolones. J Antimicrob Chemother 2000, 45(5):583-590. 
17. Fisher $L M$, Heaton VJ: Dual activity of fluoroquinolones against Streptococcus pneumoniae. J Antimicrob Chemother 2003, 51(2):463-464, author reply 464-465.

18. Fukuda H, Hiramatsu K: Primary targets of fluoroquinolones in Streptococcus pneumoniae. Antimicrob Agents Chemother 1999, 43(2):410-412.

19. Pan XS, Fisher LM: Targeting of DNA gyrase in Streptococcus pneumoniae by sparfloxacin: selective targeting of gyrase or topoisomerase IV by quinolones. Antimicrob Agents Chemother 1997, 41(2):471-474.

20. Pan XS, Fisher LM: DNA gyrase and topoisomerase IV are dual targets of clinafloxacin action in Streptococcus pneumoniae. Antimicrob Agents Chemother 1998, 42(11):2810-2816.

21. Yague G, Morris JE, Pan XS, Gould KA, Fisher LM: Cleavable-complex formation by wild-type and quinolone-resistant Streptococcus pneumoniae type II topoisomerases mediated by gemifloxacin and other fluoroquinolones. Antimicrob Agents Chemother 2002, 46(2):413-419.

22. Cambau E, Matrat S, Pan XS, Roth Dit Bettoni R, Corbel C, Aubry A, Lascols C, Driot JY, Fisher LM: Target specificity of the new fluoroquinolone besifloxacin in Streptococcus pneumoniae, Staphylococcus aureus and Escherichia coli. J Antimicrob Chemother 2009, 63(3):443-450

23. Korzheva N, Davies TA, Goldschmidt R: Novel Ser79Leu and Ser81lle substitutions in the quinolone resistance-determining regions of ParC topoisomerase IV and GyrA DNA gyrase subunits from recent fluoroquinolone-resistant Streptococcus pneumoniae clinical isolates. Antimicrob Agents Chemother 2005, 49(6):2479-2486.

24. Richter SS, Heilmann KP, Beekmann SE, Miller NJ, Rice CL, Doern GV: The molecular epidemiology of Streptococcus pneumoniae with quinolone resistance mutations. Clin Infect Dis 2005, 40(2):225-235.

25. Adam HJ, Schurek KN, Nichol KA, Hoban CJ, Baudry TJ, Laing NM, Hoban DJ, Zhanel GG: Molecular characterization of increasing fluoroquinolone resistance in Streptococcus pneumoniae isolates in Canada, 1997 to 2005. Antimicrob Agents Chemother 2007, 51(1):198-207.

26. Jorgensen JH, Weigel LM, Ferraro MJ, Swenson JM, Tenover FC: Activities of newer fluoroquinolones against Streptococcus pneumoniae clinical isolates including those with mutations in the gyrA, parC, and parE loci. Antimicrob Agents Chemother 1999, 43(2):329-334.

27. Jones ME, Sahm DF, Martin N, Scheuring S, Heisig P, Thornsberry C, Kohrer K, Schmitz FJ: Prevalence of gyrA, gyrB, parC, and parE mutations in clinical isolates of Streptococcus pneumoniae with decreased susceptibilities to different fluoroquinolones and originating from Worldwide Surveillance Studies during the 1997-1998 respiratory season. Antimicrob Agents Chemother 2000, 44(2):462-466.

28. Davies TA, Kelly LM, Pankuch GA, Credito KL, Jacobs MR, Appelbaum PC: Antipneumococcal activities of gemifloxacin compared to those of nine other agents. Antimicrob Agents Chemother 2000, 44(2):304-310.

29. Janoir C, Zeller V, Kitzis MD, Moreau NJ, Gutmann L: High-level fluoroquinolone resistance in Streptococcus pneumoniae requires mutations in parC and gyrA. Antimicrob Agents Chemother 1996, 40(12):2760-2764.

30. Munoz R, De La Campa AG: ParC subunit of DNA topoisomerase IV of Streptococcus pneumoniae is a primary target of fluoroquinolones and cooperates with DNA gyrase A subunit in forming resistance phenotype. Antimicrob Agents Chemother 1996, 40(10):2252-2257.

31. Reinert RR, Al-Lahham A, Lutticken R, Boos M, Schmitz FJ: Characterization of clinical Streptococcus pneumoniae strains from Germany with decreased susceptibility to fluoroquinolones. J Antimicrob Chemother 2002, 49(6):1015-1018.

32. Touyama M, Higa F, Nakasone C, Shinzato T, Akamine M, Haranaga S, Tateyama M, Nakasone I, Yamane N, Fujita J: In vitro activity of sitafloxacin against clinical strains of Streptococcus pneumoniae with defined amino acid substitutions in QRDRs of gyrase A and topoisomerase IV. J Antimicrob Chemother 2006, 58(6):1279-1282.

33. Lovgren M, Spika JS, Talbot JA: Invasive Streptococcus pneumoniae infections: serotype distribution and antimicrobial resistance in Canada, 1992-1995. Cmaj 1998, 158(3):327-331.

34. Clinical and Laboratory Standards Institute (CLSI): Methods for dilution antimicrobial susceptibility tests for bacteria that grow aerobically. CLSI document M7-A7. Wayne, PA; CLSI 2006.
35. Clinical and Laboratory Standards Institute (CLSI): Performance Standards for antimicrobial susceptibility testing; Sixteenth Informational Supplement. CLSI document M100-S16. Wayne, PA; CLSI 2007.

36. Davidson R, Cavalcanti R, Brunton JL, Bast DJ, de Azavedo JC, Kibsey P, Fleming C, Low DE: Resistance to levofloxacin and failure of treatment of pneumococcal pneumonia. N Engl J Med 2002, 346(10):747-750.

37. Anderson KB, Tan JS, File TM Jr, DiPersio JR, Willey BM, Low DE: Emergence of levofloxacin-resistant pneumococci in immunocompromised adults after therapy for community-acquired pneumonia. Clin Infect Dis 2003, 37(3):376-381.

38. Doern GV, Richter SS, Miller A, Miller N, Rice C, Heilmann K, Beekmann S: Antimicrobial resistance among Streptococcus pneumoniae in the United States: have we begun to turn the corner on resistance to certain antimicrobial classes?. Clin Infect Dis 2005, 41(2):139-148.

39. Orscheln RC, Johnson DR, Olson SM, Presti RM, Martin JM, Kaplan EL, Storch GA: Intrinsic reduced susceptibility of serotype 6 Streptococcus pyogenes to fluoroquinolone antibiotics. J Infect Dis 2005, 191(8):1272-1279.

40. Davies TA, Yee YC, Goldschmidt R, Bush K, Sahm DF, Evangelista A: Infrequent occurrence of single mutations in topoisomerase IV and DNA gyrase genes among US levofloxacin-susceptible clinical isolates of Streptococcus pneumoniae from nine institutions (1999-2003). J Antimicrob Chemother 2006, 57(3):437-442.

doi:10.1186/1476-0711-9-3

Cite this article as: Patel et al:: Characterization of the quinolone resistant determining regions in clinical isolates of pneumococci collected in Canada. Annals of Clinical Microbiology and Antimicrobials 2010 9:3.

\section{Publish with Bio Med Central and every scientist can read your work free of charge}

"BioMed Central will be the most significant development for disseminating the results of biomedical research in our lifetime. "

Sir Paul Nurse, Cancer Research UK

Your research papers will be:

- available free of charge to the entire biomedical community

- peer reviewed and published immediately upon acceptance

- cited in PubMed and archived on PubMed Central

- yours - you keep the copyright
BioMedcentral 